\title{
Digitalising Dentistry: A Review
}

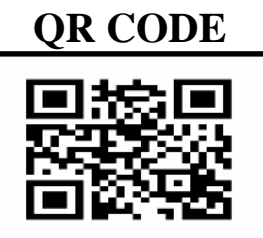

\section{NISHANT MEHTA}

Digitalisation has not left any part of our lives untouched and dentistry is no exception to it. It has revolutionised dentistry in unimaginable ways. The denture market is continously growing and advancing to better suit the patient needs. The denture or medical technology has evolved massively from its infancy stage to maturity. If you haven't visited a dentist for some time, you may be surprised to discover that there are a lot of new options to keep teeth healthy and beautiful.

KEYWORDS: Digitalisation, Dentistry, 3D printing

\section{INTRODUCTION}

The integration of technology and dentistry has given mind-blowing results. The technology and the armamentarium with the patient's need has been interwoven to achieve the highest success Rates. It brings many advantages on the table for the dentist, the assistant and the patient. Technology has not only uplifted the accuracy and intricacy of dentistry but has reduced the burden on the clinician as well. The astounding thing about dental technology is how much it lends from other industries-CAD/CAM came from the manufacturing sector, and it just took some software tweaks to improve the accuracy.

Traditionally, case history and physical examination, two-dimensional X-ray data radiology (periapical, panoramic, and cephalometric radiographs), was the backbone for the necessary preparatory stages for formulating a treatment plan and for carrying out the therapy. With only twodimensional X-ray data available, establishing correct diagnosis and an appropriate treatment plan could be arduous; therapies essentially relied on the manual skills and experience of the operator. ${ }^{1}$

But now, Digitalisation comes with bounty of blessings. The speed and simplicity of procedures, which means the patient can be in and out of the clinic with their teeth completely restored in way less time than with conventional techniques. Temporisation and never ending repeat appointments are a thing of past now, dentistry has revolutionised the patient experience. Single appointment techniques with the ability to diagnose, create and fit the restoration has made dentistry more fit for the 21st century. One can enter and access big data just at the touch of our fingers.

\section{THE DIGITALIZATION OF HEALTH RECORDS}

With the advent of technology keeping medical records, history, vaccination records became less tedious and simpler than ever. EHRs or Electronic Health Records are replacing paper records have benefited all medical healthcare professionals from doctors, nurses, dentists, medical records and information technicians. It has also simplified medical billing with its implementation.

Public health: It has immensely helped in the field of public health as it is easier for the health researchers to access the data through EHRs. It has also aided in diagnosing diseases and treating them with a central standardised system. The outbreak of viruses or infections- their clinical features and treatment modalities, prevention protocols are all uploaded into the system which helps in containing them which would otherwise result in epidemics.

Better care for the patient: in case of an unconscious patient, being able to retrieve their medical history through EHRs can prove to be lifesaving, since it gives us information regarding underlying diseases, on-going medications, drug allergies.

Simplified workflow: Medical billers and coders are perhaps most impacted by EHRs, as the number of medical codes recently jumped from 13,600 to 
69,000. The introduction of EHRs has made life for medical billers and coders much easier despite this huge jump. ${ }^{2}$

Communication advancements: Almost every person owns a cell phone today, and is connected to the internet. This has helped reach people in more remote areas where a specialist cannot reach. For dentists, there are video call appointments to diagnose and devise a treatment plan when the patient and the dentist are in different countries. Technology has also been used to educate the masses. With the rise in telemedicine, the concept of medical assistants emanated. The dentist communicates with the assistant over a call or video conferencing and the medical assistant maintains records while sitting at the other end of the world. This has drastically reduced the waiting time for patients.

Teledentistry: Teledentistry comprises of a computer with hard drive memory, enough RAM, and a fast processor, an intraoral video camera or a digital camera for the capture of picture, a modem and an internet connection. Other additions to the unit can be a fax machine, a scanner, and a printer. ${ }^{3}$ During the consultation, the patient is not present. Dentists can share patient information, radiographs of periodontal and hard tissues, therapies applied lab results, tests, remarks, photographs, and other information retrieved through multiple providers. This data sharing can be of paramount importance for patients, especially those in need of specialist consultation. (4) As India is an agrarian nation and most of the population belongs to rural areas where some of the basic amenities of daily life are deficient, especially primary health education and oral health care services. In order to provide better services to the population, Primary Health Centre and Community Health Centre can be equipped with modern telecommunication systems for referral. Also, the Ministry of Health and Family Welfare Government of India has launched the power of mobile for Public Health in India on 2oth January 2016.4

CAD-CAM: CAD/CAM technologies have started a new age in dentistry. The quality of dental prostheses has enhanced significantly by means of these production processes. This makes very efficient quality management possible. On the one hand it increased the productivity drastically and changed dental laboratories from manufacturers to modern computerised production units. On the other hand this increase in productivity leads to a competitive capability to produce dental prostheses independent of the manufacturing site, which might be a major factor for the high wage countries to keep business volume in the country. Last but not least CAD/CAM technology has made it possible to use the new materials like the high performance ceramics and titanium with high accuracy and intricacy. ${ }^{5}$

The presently available software of CAD/CAM systems on the market is being constantly improved. The latest construction possibilities are continuously available to the user at the touch of their fingers with new updates. The storage of this data can be in various formats and forms. The basis therefore is often standard transformation language (STL) data. ${ }^{6}$

\section{THE MOST RECENT ADVANCES IN DENTISTRY}

Digital dentistry and ${ }_{3} \mathrm{D}$ printing: These printers accurately help in the production of models which aid in diagnosis and treatment planning. They are affordable, reliable and scalable. Their application is wide, for example: Dental and Orthodontic Models

\section{Castable Crowns, Bridges, Copings and Partial Denture Frameworks: \\ - Direct Crowns and Bridges (E-Dent 40o) \\ - Bite Splints or Night Guards (E-Guard) \\ - Indirect Bonding Trays (E-IDB) \\ - Surgical Drill Guides (Clear Guide and E- Guide Tint) \\ - Flexible Gingiva Masks (E-Gum) \\ - $\quad$ Denture Bases (E-Denture) ${ }^{7}$}

These devises have eliminated the time consuming, tideous processes and also the scope of error. The conventional techniques included taking impressions with materials which were nto as conveinent for the patint as the scanners.This has been particularly beneficial to the patients with gag reflex.

Superior visualization: After a lot of research and inventions, today we have products in the market 
which can magnify what a normal human eye can see and hence prove the accuracy and precision of the procedures. OmniOptic is one such product, it supports four magnification powers from 2.5 times to 5.5 times within the same loupe system. ${ }^{8}$

Dentistry with the "Wand": The wand is a devise which is used to deliver anaesthesia in a slow painless manner. This devise also prevents collateral numbness. Air Abrasion systems have been implicated in treating small cavities, repair older restorations, removing stains and discolorations.

Advances in Implantology: Implants have gained popularity in the past decade. Implant placement is a technique sensitive procedure and failure of an implant is detrimental for future prosthetic treatment modalities. The applications of this technology initially were limited to toothsupported restorations. However, implant dentistry has accepted this technology in a very enthusiastic way owing to no casting shrinkage, greater passivity of prosthesis, and ability to mill titanium that results in very light weight prosthesis. The field of maxillofacial prosthodontics is also using different software to design and wax mill the prosthesis before silicon duplication. ${ }^{9}$

A recent introduction of a new solution to tackle a fixed-hybrid implant failure called as the Locator FTx Fixed Attachment System, and is claimed as "the only immediate treatment solution that can rescue a patient's existing fixed-hybrid prosthesis after replacing an implant and maintain a passive fit."

This allows the dentists to fix instead of replacing the prosthesis. The patient benefits from an immediate solution and also save money by avoiding the longer, tideous more involved replacement process. ${ }^{8}$

HD pictures: A dentist has plethora of options to choose his digi-cam companion from. They come with wide variety of features, chemical and water resistant. High detailing in pictures aids the dentist to make better decisions for the patient. Cameras have become one good investment for a dental clinic.

Rapid Prototyping: Rapid prototyping (RP) is the customized production of solid models using $3 \mathrm{D}$ computer data. Over the past decade, there have been so many advances in RP, resulting in the development of new techniques that have been applied to the fabrication of various prostheses. To name a few RP technologies there is stereolithography (SLA), fused deposition modelling (FDM), computer numerical controlled (CNC) milling, and, more recently, selective laser sintering (SLS). The applications of RP techniques for dentistry is vast like wax pattern fabrication for dental prostheses, dental prostheses mold fabrication, and removable dental prostheses framework fabrication. This new approach has also been reducing labour-intensive laboratory procedures can generate the wax pattern for facial prostheses directly. Many people stand to benefit from these new RP techniques for producing various forms of dental prostheses, which in the near future could transform traditional prosthodontic practices. ${ }^{10}$

\section{The First Dual-Wavelength Soft Tissue Diode Laser:}

The diode laser is a dual-wavelength soft tissue diode laser, as well as the most powerful soft tissue laser available to dentists, which can be utilised with both wavelengths simultaneously. It combines the optimal melanin absorption of an 810 nanometer wavelength laser with the optimal water absorption of a 980 nanometer wavelength laser. This laser makes every process faster, smoother, and more efficient. ${ }^{11}$

A Bluetooth for a toothbrush: These days every devise comes equipped with bluetooth, toothbrushes aren't far behind. They also have inbuilt bluetooth which help take better care of teeth and gums. They have pressure sensors which prevent you from brushing too hard or too lightly, the more caries prone areas in the mouth are highlighted in the $3 \mathrm{D}$ mouth apps, also the missed spots while brushing are highlighted.

Caries Detection: A new caries detector has been launched which aid in caries and crack detection. It is a portable device which runs on trans illumination technology.

Dental impressions: Intra-oral scanners scan the teeth and gums quickly and with no discomfort to the patient while maintaining the accuracy that promises better treatment and diagnosis. Such 
scanners have decreased the patient discomfort associated with conventional impression techniques.

\section{CHALLENGES IN DIGITAL DENTISTRY}

There is a large gap between the technician and the dentist. The technicians have been using digital techniques for long now but not all dentist have welcomed these new techniques as they still take impressions, make casts and physically deliver them. In order to fully utilise the true potential of digitalisation drift, the dentists also need to the start using more of these techniques.

Furthermore, the use of this technology needs skill and precision of the clinician although they are to simplify the workflow. They can work wonders only in the hand of a skilled person and can bear disastrous results if not used aptly. The digital methods do not allow any room for mistakes, unlike the conventional techniques which can be easily fixed. The best way to overcome this shortcoming is use these techniques during the early education years if dentistry, their use should be incorporated in the curriculum.

To conclude, the future of digital dentistry is bright and has a lot of scope but it does come with its shortcomings. Although these shortcomings can easily be overcome. In the coming years digital dentistry will make the lives of the patient and dentist a lot easier and happier.

\section{REFERENCES}

1. Mangano F, Shibli JA, Fortin T. Digital Dentistry: New Materials and Techniques. International Journal of Dentistry. 2016;2016:5261247.

2. https://www.aimseducation.edu/blog/theimpact-of-technology-on-healthcare/ [Accessed on $21^{\text {st }}$ January, 2018]

3. Birnbach JM. The Future of Teledentistry. J Calif Dent Assoc28, 2000, 141-3.

4. Gupta B, Gupta N, Thakar S, Gupta P, Jindal S. Dentistry on web-teledentistry. Int J of Allied Med Sci and Clin Res 2017; 5(1): 196-200.

5. Beuer, Florian \& Schweiger, Josef \& Edelhoff, Daniel. (2008). Digital dentistry: An overview of recent developments for CAD/CAM generated restorations. British dental journal. 204. 505-11.

6. Mehl A, Gloger W, Kunzelmann K H, Hickel R. A new optical 3-D device for the detection of wear. J Dent Res 1997; 76: 1799-807.

7. https://envisiontec.com/3d-printingindustries/ medical/dental/ [Accessed on $15^{\text {th }}$ January, 2018]

8. https://www.tiogadental.com/dental-technology2018/ [Accessed on 25 ${ }^{\text {th }}$ January, 2018]

9. Parkash H. Digital dentistry: Unraveling the mysteries of computer-aided design computeraided manufacturing in prosthodontic rehabilitation. Contemporary Clinical Dentistry. 2016;7(3):289-9o.

10. Begum Z, Chheda P. Rapid Prototyping - When Virtual meets Reality. International journal of computerized dentistry 2014;17:297-306.

11. https://www.ultradent.com/SiteCollection

Images/Multi-Media-Tab/Brochures/Equipment/

Documents/Europe/UPP\%20510-EU Gemini

Brochure.pdf [Accessed on $20^{\text {th }}$ January, 2018]

Source of support: Nil, Conflict of interest: None declared

Cite this article as:

Mehta N. Digitalising Dentistry: A Review. Int Healthcare Res J 2018;2(4):82-85 doi: 10.26440/IHRJ/02_04/180

\section{AUTHOR AFFILIATIONS:}

Senior Resident, Unit of Public Health Dentistry, Oral Health Sciences Centre, PGIMER, Chandigarh

Corresponding Author:

Dr. Nishant Mehta

Senior Resident, Unit of Public Health Dentistry

Oral Health Sciences Centre, PGIMER, Chandigarh
For article enquiry/author contact details, e-mail at: manuscriptenquiry.ihri@gmail.com 